\title{
COMMISSION 25: STELLAR PHOTOMETRY AND POLARIMETRY
}

Report of Meetings, 26, 27, 29 July 1991

PRESIDENT: Ian S. Mclean

SECRETARY: E. Milone

\section{July 1991}

The Business Meeting of Commission 25 at the XXI IAU General Assembly was held during Session 2 on July 26, 1991 in Room G, San Martin Cultural Center. The President Ian McLean called the meeting to order, welcomed members and attendees, and requested approval of the Agenda for the meeting. With the Agenda approved by the members present, Prof. McLean reported on changes in membership. There had been 2 resignations and 20 new proposed Comm 25 members ( 14 at the GA), giving a total new membership of 200 . There were no objections to any of the proposed new members. The President reported that 2 IAU Symposia (Nos. 143 and 148) had been supported and 3 Colloquia were being sponsored by Comm. 25. Also, a half-yearly Newsreport had been initiated. He reviewed the triennial report and distributed copies.

\section{Elections}

Voting on new officers was carried out. New Organizing Committee members are: S. Adelman, R. Genet, T. Moffett, A. Landolt and C. Sterken. Continuing to serve are: M. Breger, I.K. Knude; J.D. Landstreet, J. Lub, J. Menzies, V. Straizys, F.J. Vrba and the retiring president I.S. McLean. The President placed the name of John Landstreet (not present) in the nomination for Vice-President. Landstreet was elected unanimously. As is the custom, Dr. A.T. Young our current Vice-president was nominated for President, Dr. M. Breger seconding the nomination. Andy was was elected by a unanimous vote.

\section{Discussion on the future of the Commission}

The President invited discussion on the future of the commission, stating that the IAU had requested that commissions reappraise their role, so that consolidation of some commissions could be carried out. A spirited discussion among the members ensued with the conclusion being that we desire to continue as a commission and have the arguments to defend our position.

\section{New Business}

Working groups: Landstreet is a representative on a W.G. on Ap stars. Another multi-commission WG is the one on synthetic photometry; Comm 25 is represented by Buser. A small W.G. on the infrared was formed at the last GA by Milone; input to this working group would be welcome. Garrison is responsible for standard stars and Chris Corbally is the new newsletter chairman in this WG. Sterken: Important questions which we need to consider are how many and how faint. Garrison: Standardization of filter systems could be included in the newsletter. Crawford: Said dialogue on these matters is important and MOVED that a small W.G. be formed to report back in three years. A.T. Young will coordinate. 


\section{$\underline{27, \text { July } 1991}$}

\section{PHOTOMETRY SESSION: Chair - I.S. McLean}

In the absence of any of the authors of the report on the Vilnius system, A.T.Young read the short note "A Possibility of Photometric Three-dimensional Classification of Stars at V=20" by V. Straizys, F. Smriglio, and R. P. Boyle. The 7-band Vilnius system allows photometric determination of spectral class, absolute magnitude, and metallicity, regardless of interstellar reddening, and permits recognition of many types of stellar peculiarities (Straizys, "Multicolor Stellar Photometry"; Pachart, 1990).

C. Sterken discussed "The future of existing photometric systems." Every new detector brings more systems, incompatible with the old ones. Standardization is very difficult. Problems connected with colors remain unsolved since the 1920's. It is difficult to escape from these problems for many reasons. We must return to teaching fundamentals of photometry to all observers, develop standard reduction programs and we need a new standard system that can be reproduced accurately; it must be well-calibrated, and "open". An approach is to buy many identical filter sets and install them in a world-wide network of APTs.

A. Landolt reported on his UBVRI (Cousins RI) standards to V=16 and 21. Extinction was measured every night at CTIO; though he reported mean values, the night-to-night variations are clear and significant. He has observed 20 to 30 standard stars per night. An interesting finding is that the RMS error changes considerably with time during the night. Of 526 candidate stars, 298 survived as standards, observed about 29 times each. The transformations are nonlinear. Typical mean errors of the final means are 3 millimags. The response functions used will be published, both for filters and for the PMTs used.

A. T. Young then discussed "Transformations and accuracy: problems and possibilities." Menzies et al. (MN 248, 642, 1991) find systematic differences of several hundredths of a magnitude from Landolt, in measuring the Landolt equatorial standards in the Cousins version of UBV. These problems are basically aliases, because the bands under-sample the spectrum. The problems lie in the fundamentals of photometry. The Stromgren-King theory uses a Taylor-series expansion to obtain terms that are products of moments and derivatives (Stromgren 1937, in "Handbuch der Experimentalphysik"; King 1952 in AJ 57, 253). It turns out that the derivatives increase as fast as the moments decrease when passband width changes; the terms maintain their relative size, and only the factorials in the denominators allow convergence. Terms of high order MUST be retained. Accurate transformations should be possible if bands that sample the spectrum correctly are used.

E. F. Milone reported status of infrared photometry: There have been a few developments in IR systems since the meeting on Infrared Extinction and Standardisation [A volume was published by Springer Verlag in the series 'Lecture Notes in Physics', No. 341, 1989. ] (hereafter IRES) at the Baltimore General Assembly. In system definition and transformation: Bessell and Brett (summary in IRES, pp. 61, 1989; details in PASP, 100, 1134, 1988) review previous work and compare several JHKLL'M systems in use at that time (SAAO, ESO, CIT/CITO, MSO, AAO, and Arizona), and derive linear transformation coefficients and zero points among them. They also proposed a "homogenized" system, the MSO JHKL system. Establishment of a standard system involving fainter stars is an increasingly important need (McLean, IRES, p. 66, 1989). A working 
group led by Milone is looking at defining a new system ; all interested parties are urged to contact and perhaps join the working group.

\section{July, 1991}

\section{CONTRIBUTIONS OF POLARIMETRY TO STELLAR ASTROPHYSICS}

(A joint meeting with Commissions 9, 27, and 42)

Chair: Bob Koch (morning), Ian McLean (afternoon)

The following papers were presented:

\section{A review of physical processes - K. Nordsieck}

From periodic and non-periodic variability of $Q$ and $U$ one can ascertain information such as inclination, axis orientation, sense of rotation, location of scatterer. Spectropolarimetry can separate different kinds of scattering by wavelength dependence e.g. electron, Rayleigh, and Mie scattering. For example, nebular polarization is strongly depolarized by light from the central star: ie. PMS stars seen only in scattered nebular light have big polarizations, those seen directly have much smaller polarizations. When $\tau \ll 1$ the problem is that the dust envelope is poorly constrained whereas for $\tau \gg 1$ the problem is that radiative transfer is not trivial.

Array detectors in astronomical polarimetry - J. Tinbergen and $\underline{\mathrm{I} . \mathrm{McLean}}$

After first generation of polarimeters, area detectors have given a major advance - CCDs being the best of these. But the problem is how to do rapid modulation when the CCD readout is normally slow. One solution is to split $E$ and $O$ rays, image them on different pixels, and find $Q$ and $U$ from the ratio. Current $C C D$ and IR polarimeters were reviewed and details were given of the ISIS spectropolarimeter for the 4.2-m W.H.T.

Interstellar polarization in the environments of star forming regions - F.J. Vrba, H.G. Marraco, S.E. Strom, L. Hillenbrand. For Herbig Ae/Be stars we have obtained new optical and infared photometry, new polarimetry of the stars and surrounding local ISM, and determined jet/axial rotation directions from optical and molecular mapping and disk models from the literature. The resultant Spectral Energy Distributions group into two: those that follow a wavelength to the $-4 / 3$ power (indicative of optically thick reprocessing or accretion disks) or those with flat or rising SEDs (indicative of the presence of material outside of the accretion disk). The results support the view that YSO polarizations are due to scattered light rather than aligned grains in a disk.

\section{Problems with polarization standards - J. Dolan}

For the high polarization standards used for position angle (PA) calibration, it is difficult to do better than about $\sigma=1^{\circ}$. To get $\sigma<1$ degree, we need that the PA is NOT a function of time or wavelength, although the latter is OK if bandpasses are well-defined. The problem with high polarization stars is that they are typically distant giants with a component of intrinsic polarization due to scattering from extended envelopes. What to do about this?

a) observe asteroids since scattering plane between Earth-asteroid-Sun is well-determined

b) mechanically align Glan-Thompson prism to N-S, can be done to 0.1 degree if careful.

A new polarization study of the magnetic Babcock's star - M. Breger and N.S. Polosukhina Data in literature appear to show variable polarization for this star, but not when you take into account error bars. New UBVRI polarimetric observations show no variability and that observed 
polarization is completely consistent with ISM polarization.

Spectropolarimetry of massive hot stars - R. Schulte-Ladbeck

Summary of results: EZ CMa - P and theta changes correlated with emission line flux. HD 191765 - $\mathrm{P}$, but not theta correlated eith emission line flux. If $\mathrm{P}($ line) not same as $\mathrm{P}$ (cont) this implies intrinsic polarization and the quantities $\mathrm{P}($ line $) / \mathrm{P}(\mathrm{cont})$, deltaQ, and deltaU reveal information about atmospheric structure and winds. Polarized lines are slightly red-shifted which implies outflowing winds.

WUPPE results: hot unevolved and evolved objects - $\mathrm{K}$. Nordsieck

Zeta Tau - Be star w/ constant polarization - line blanketing w/ Balmer jump in polarization.

Pi Aqr - Be star w/ variable polarization - line blanketing but w/o Balmer jump in polarization.

Kappa Cas - hot supergiant - only interstellar polarization. Alpha Cam - hot supergiant - only interstellar polarization. P Cyg - hot supergiant - high states of polarization during WUPPE obs Alpha Ori - cool supergiant - see polarization features coincident with TiO bands - actually 2 sets at 45 degree different theta - (a) narrow TiO theta, (b) at blue upturn theta. Also see Mg II 2800 chromospheric lines in polarization at above thetas. Far UV results imply process in atmosphere not in circumstellar shell.

Environments of luminous late-type variables - A.M. Magalhaes

L2 Pup - non-uniform stellar disk, grain size changes during course of years, stable symmetry plane from IR observations: V CVn - P anti-correlated with brightness: o Ceti - optical p(lambda) closely related to shockwave, $P$ increases until shock dies out.

Miras imaged by speckle and their polarization signatures - R. Boyle

Mira A and B studied with polarimetric coverage during 4 different epochs between Nov 1983 and Dec 1988. Before phase 0.8 (eruption point) $P$ slowly decreases, after phase $0.8 \mathrm{P}$ suddenly grows with sudden increase of grains in shell. Dust located at about 3 stellar radii by 11 micron spatial interferometry at $\mathrm{T}=1200 \mathrm{~K}$. The radius depends on wavelength $(0.030-0.100 ")$; outside of TiO bands it is typically $0.04 "$.

Polarization of young stellar objects: general properties and a few examples - P. Bastien Unresolved observations of $\mathrm{T}$ Tauri, Herbig Ae/Be stars yield no standard polarization lambda dependence, P can be large: e.g. HL Tau 12\%, V376 Tau 21\%. Stars with IR excesses have larger $\mathrm{P}$ and observed circular polarization implies dust not electron-scattering. Resolved observations reveal extended, centro-symmetric polarization pattems so you can find the illuminating source. Reported 56 galactic sources with polarization maps (mostly YSOs): $20 \%$ centro-symmetric, $60 \%$ aligned vectors and $11 \%$ peculiar. Multiple scattering is the cause - not aligned grains.

\section{Scattering envelopes in Wolf-Rayet stars - A. Moffat}

16 early-type binaries with polarization observed - of these 11 are Wolf-Rayet stars.

There are two types of polarization: (a) No obvious periods in polarization - great deal of variability in many cases, in other cases no variability or even any polarization - implies winds are symmetric (b) Periodic polarization variability - due to binary modulation - sometimes modes a) and b) are seen together. Interpretation - Polarization is produced by electron-scattering with sufficient asymmetry to produce polarization. From these observations one can get orbits. Several circular and eliptical orbits have now been fit. 